\title{
On the Boundary Value of a Solution of the Heat Equation
}

\author{
By \\ Takahiro KAWA* and Tadato MATSUZAWA**
}

\section{$\S 0$. Introduction}

Let $U$ be an open subset of $\mathbf{R}^{n}$, and let $P\left(x, D_{x}\right)$ be a linear differential operator with analytic coefficients defined on a neighborhood of the closure $[U]$ of $U$. Suppose that the boundary $\partial U$ of $U$ is smooth (i.e., non-singular and analytic) and that $\partial U$ is non-characteristic with respect to $P$ at each point in $\partial U$. Then it is well-known $([7],[11])$ that the boundary value of a hyperfunction solution of the equation $P u=0$ on $U$ is a well-defined hyperfunction. However, little is known about the characterization of a solution whose boundary value determines a hyperfunction near a characteristic boundary point. The purpose of this article is to discuss this problem for one special case, i.e., the pair of the heat operator $\partial / \partial t-\Delta \underset{\text { def }}{\partial} \partial / \partial t-\sum_{j=1}^{n-1} \partial^{2} / \partial x_{j}^{2}$ and the domain $\left\{(t, x) \in \mathbb{R}^{n} ; t>0\right\}$. Our main result (Theorem 1 below) asserts that, and

(i) if a $C^{\infty}$-solution $u(t, x)$ does not behave too wildly as $t \downarrow 0$,

(ii) if $u(t, x)$ uniformly tends to zero outside a compact set $K \subset \mathbf{R}_{x}^{n-1}$ as $t \downarrow 0$,

then we can assign a compactly supported hyperfunction $g(x)$ to $u(t, x)$ so that the vanishing of $g(x)$ entails the vanishing of $u(t, x)$ itself. Furthermore we can find such a tame solution $u(t, x)$ of the heat equation for any compactly supported hyperfunction $g(x)$. [See Theorem 1 for the precise statement. Note also that a hyperfunction supported by a compact set, say $L$, is an analytic functional with the real carrier $L$.]

Let us note the following two facts: First, if $u(t, x)$ tends to infinity too rapidly as $t \downarrow 0$, then our procedure will not assign a hyperfunction $g(x)$. (Cf. §2(i)) Second, we know (see [3], for example) that there exists a hyperfunction $e(t, x)\left(x \in \mathbf{R}^{1}\right)$ supported by $\left\{(t, x) \in \mathbb{R}^{2} ; t=0, x \geqq 0\right\}$ satisfying the equation

Received December 1, 1988.

* Research Institute for Mathematical Sciences, Kyoto University, Kyoto 606, Japan.

** Department of Mathematics, Nagoya University, Nagoya 464, Japan. 
$\left(\partial / \partial \mathrm{t}-\partial^{2} / \partial x^{2}\right) e(t, x)=\delta(t) \otimes \delta(x)$. Putting this differently, we claim that there exists no reasonable assignment of a hyperfunction $g(x)$ to $u(t, x)$ if the condition (ii) is not satisfied.

We would like to express our heartiest thanks to Professor Mikio Sato for the stimulating discussion with him.

\section{$\S 1$. Main Results}

To state our main result (Theorem 1 below) we first introduce the following symbol:

For a compact subset $K$ of $\mathbb{R}^{n-1}$, we denote by $\mathscr{S}_{K}^{\text {tame }}$ the totality of $C^{\infty}$ solutions of the heat equation $(\partial / \partial t-\Delta) u(t, x)=0$ on $\left\{(t, x) \in \mathbb{R}^{n} ; t>0\right\}$ that satisfy the following condition:

(1) For each $\varepsilon>0$, there exists a constant $C_{\varepsilon}$ for which

$$
|u(t, x)| \leqq C_{\varepsilon} \exp \left(\frac{\varepsilon}{t}-\frac{\operatorname{dist}(x, K)^{2}}{4 t}\right)
$$

holds on $\left\{(t, x) \in \mathbb{R}^{n} ; t>0\right\}$.

Here dist $(x, K)$ denotes the distance of the point $x\left(\in \mathbb{R}^{n-1}\right)$ and $K$.

Theorem 1. Let $K$ be a compact subset of $\mathbb{R}^{n-1}$ and let $\mathscr{B}_{K}$ denote the space of $(n-1)$-dimensional hyperfunctions supported by $K$. Then there exists an isomorphism $b: \mathscr{S}_{\mathrm{K}}^{\text {tame }} \rightarrow \mathscr{B}_{\mathrm{K}}$. Furthermore, for each $u$ in $\mathscr{S}_{\mathrm{K}}^{\text {tame}}$, we can find a hyperfunction $\tilde{u}(t, x)$ satisfying $\left.\tilde{u}\right|_{\{t>0\}}=u$ and $\operatorname{supp} \tilde{u} \subset\left\{(t, x) \in \mathbb{R}^{n} ; t \geqq 0\right\}$ so that the following holds:

$$
(\partial / \partial t-\Delta) \tilde{u}(t, x)=\delta(t) \otimes b(u)
$$

Remark. Since supp $\tilde{u} \subset\{t \geqq 0\}$, the relation (2) implies that $b(u)$ describes the "boundary value" of $u$ taken from the side $\{t>0\}$. Hence Theorem 1 guarantees the existence of the "boundary value" of $u$ as a hyperfunction on the condition that the solution $u$ in question satisfies the condition (1), although the boundary $\{t=0\}$ is characteristic with respect to the heat equation.

Proof. Our construction of the map $b$ uses a particular extension of $u$ to the entire space $\mathbb{R}^{n}$. To define such a preferred extension, let us first introduce an auxiliary set $\Omega=\left\{(t, x) \in \mathbb{R}^{n} ; t \neq 0\right.$ or $\left.x \notin K\right\}$. By using the classical estimate for solutions of heat equations (see [2], for example), we find that the condition (1) implies

$$
\lim _{t \downarrow 0} P(\partial / \partial t, \partial / \partial x) u(t, x)=0 \quad \text { for } x \notin K
$$

for any linear differential operator (with constant coefficients) $P(\partial / \partial t, \partial / \partial x)$ of finite order. Hence we can find a $C^{\infty}$-function $c(t, x)$ which satisfies the following conditions: 
(4) $c(t, x)=u(t, x)$ if $t>0$,

(5) $c(t, x)$, together with all its derivatives, vanishes on $\left.\Omega \backslash\{t, x) \in \mathbb{R}^{n} ; t>0\right\}$. [What we actually need in the subsequent reasoning is not the $C^{\infty}$-character of $c$ but its $C^{2}$-character.] Since the assumption (1) implies that $u(t, x)$ does not increase faster than $\exp (\varepsilon / t)$ as $t \downarrow 0$ for any $\varepsilon>0$, a result of Komatsu ([6]), Theorem 2.27) guarantees the existence of an ultradistribution $v(t, x) \in \mathscr{D}^{\{2\}}\left(\mathbf{R}^{n}\right)$ which satisfies the following:

$$
v=c \text { on } \Omega .
$$

See [4] for the definition and basic properties of the space $\mathscr{D}^{\{2\}}\left(\mathbf{R}^{n}\right)$, the space of ultra-distributions of Gevrey-type $\{2\}$.

Since $c(t, x)$ vanishes for $t<0$, we find

$$
\operatorname{supp} v \subset\left\{(t, x) \in \mathbb{R}^{n} ; t \geqq 0\right\} .
$$

Further (4) and (5) imply

$$
(\partial / \partial t-\Delta) c=0 \quad \text { on } \Omega
$$

and hence

$$
(\partial / \partial t-\Delta) v=0 \quad \text { on } \Omega .
$$

In what follows, an ultradistribution $v(t, x)$ thus obtained shall be called a tame extension of $u$ for short.

Now, letting $\mu(t, x)$ denote $(\partial / \partial t-\Delta) v$ we find the following:

$$
\operatorname{supp} \mu \subset\left\{(t, x) \in \mathbb{R}^{n} ; t=0, x \in K\right\} .
$$

Since $\mu$ belongs to $\mathscr{D}^{\{2\}^{\prime}}\left(\mathbb{R}^{n}\right)$, a structure theorem for ultradistributions supported by a submanifold ([5]), entails the following:

$$
\mu(t, x)=\sum_{k=0}^{\infty} \delta^{(k)}(t) \otimes \mu_{k}(x)
$$

where $\delta^{(0)}(t)=\delta(t), \quad \delta^{(k)}(t)=\partial^{k} / \partial t^{k} \delta(t) \quad(k=1,2, \cdots) \quad$ and $\quad \mu_{k}(x) \in \mathscr{D}^{\{2\}}\left(\mathbb{R}^{n-1}\right) \quad$ is supported by $K$ and satisfies the following condition:

(12) For any strictly positive constants $L, h$ and $\delta$, there exists a constant $C$ for which

$$
\left|\mu_{k}(\varphi)\right| \leqq C L^{k}(k !)^{-2} \sup _{x \in K_{\delta, \alpha}} \frac{\left|D^{\alpha} \varphi(x)\right|}{h^{|\alpha|}(\alpha !)^{2}}
$$

holds for any $\varphi$ in $\mathscr{D}^{\{2\}}\left(\mathbb{R}^{n-1}\right)$.

Here, and in what follows, $\alpha=\left(\alpha_{1}, \ldots, \alpha_{n-1}\right)$ is a multi-index with $\alpha_{j}$ being a nonnegative integer, $|\alpha|$ denotes $\sum \alpha_{j}$, and $K_{\delta}=\left\{x \in \mathbb{R}^{n-1}\right.$, dist $\left.(x, K) \leqq \delta\right\}$.

Let us now set 


$$
Q_{k}=\sum_{\ell=0}^{k-1} \partial^{\ell} / \partial t^{\ell} \Delta^{k-1-\ell}
$$

for $k=1,2, \cdots$. Here and in what follows, $\partial^{0} / \partial t^{0}$ and $\Delta^{0}$ denote the identity operator. Then we find

$$
\left(\partial^{k} / \partial t^{k}-\Delta^{k}\right)=(\partial / \partial t-\Delta) Q_{k}
$$

for $k=1,2, \cdots$. It follows from (12) that both $\sum_{k=1}^{\infty} Q_{k}\left(\delta(t) \otimes \mu_{k}(x)\right)$ and $\delta(t) \otimes\left(\sum_{k=0}^{\infty} \Delta^{k} \mu_{k}(x)\right)$ belong to $\mathscr{B}_{\{0\} \times K}$, the space of hyperfunctions supported by $\{0\} \times K$. Denoting $\sum_{k=1}^{\infty} Q_{k}\left(\delta(t) \otimes \mu_{k}(x)\right)$ and $\sum_{k=0}^{\infty} \Delta^{k} \mu_{k}$ respectively by $v(t, x)$ and $g(x)$, we obtain the following equality (15) from (14):

$$
(\partial / \partial t-\Delta)(v-v)=\delta(t) \otimes g(x) .
$$

Let us next verify $g(x)$ thus defined is independent of the choice of a tame extension $v$. Let $v_{1}$ and $v_{2}$ be two tame extensions of $u$. Then it follows from the definition of the tame extension that $v_{1}-v_{2}$ vanishes on $\Omega$, i.e.,

$$
\operatorname{supp}\left(v_{1}-v_{2}\right) \subset\{0\} \times K .
$$

Since both $v_{1}$ and $v_{2}$ belong to $\mathscr{D}^{\{2\}^{\prime}}\left(\mathbb{R}^{n}\right)$, we can find

$$
h_{k}(x) \in \mathscr{D}^{\{2\}^{\prime}}\left(\mathbb{R}^{n-1}\right) \quad(k=0,1,2, \cdots)
$$

which satisfy the following:

$$
\begin{gathered}
\operatorname{supp} h_{k} \subset K \text { for } k=0,1,2, \cdots \\
v_{1}-v_{2}=\sum_{k=0}^{\infty} \delta^{(k)}(t) \otimes h_{k}(x)
\end{gathered}
$$

(19) For any strictly positive constants $L, h$ and $\delta$, there exists a constant $C$ for which

$$
\left|h_{k}(\varphi)\right| \leqq C L^{k}(k !)^{-2} \sup _{x \in K_{\delta}, \alpha} \frac{\left|D^{\alpha} \varphi(x)\right|}{h^{|\alpha|}(\alpha !)^{2}}
$$

holds for any $\varphi$ in $\mathscr{D}^{\{2\}}\left(\mathbb{R}^{n-1}\right)$.

Let $\rho_{k}(x)$ denote the difference of $\mu_{k}$ 's determined by $v_{1}$ and $v_{2}$ respectively, that is,

$$
\sum_{k=0}^{\infty} \delta^{(k)}(t) \otimes \rho_{k}(x)=(\partial / \partial t-\Delta)\left(v_{1}-v_{2}\right)
$$

Since

$$
(\partial / \partial t-\Delta)\left(v_{1}-v_{2}\right)=(\partial / \partial t-\Delta)\left(\sum_{k=0}^{\infty} \delta^{(k)}(t) \otimes h_{k}(x)\right)
$$




$$
=\sum_{k=0}^{\infty}\left(\delta^{(k+1)}(t) \otimes h_{k}(x)-\delta^{(k)}(t) \otimes \Delta h_{k}(x)\right),
$$

the comparison of the coefficients of $\delta^{(k)}(t)$ in (20) shows

$$
\begin{gathered}
\rho_{0}(x)=-\Delta h_{0}(x), \\
\rho_{k}(x)=h_{k-1}(x)-\Delta h_{k}(x) \quad \text { for } k=1,2, \cdots .
\end{gathered}
$$

Therefore we find

$$
\begin{aligned}
\rho_{0}+ & \sum_{k=1}^{\infty} \Delta^{k} \rho_{k} \\
& =-\Delta h_{0}+\sum_{k=1}^{\infty} \Delta^{k} h_{k-1}-\left(\sum_{k=1}^{\infty} \Delta^{k+1} h_{k}\right) \\
& =\sum_{k=1}^{\infty} \Delta^{k} h_{k-1}-\left(\sum_{\ell=1}^{\infty} \Delta^{\ell} h_{\ell-1}\right)=0
\end{aligned}
$$

This means that $g(x)$ does not depend on the choice of a tame extension $v$ of $u$. Hence we define $b(u)$ by $b(u)=g$. Since the sum of tame extensions $v_{j}(j$ $=1,2)$ of $u_{j}\left(j=1,2\right.$, respectively) in $\mathscr{S}_{K}^{\text {tame }}$ is a tame extension of $u_{1}+u_{2}, b(u)$ thus defined is a linear map from $\mathscr{S}_{K}^{\text {tame }}$ into $\mathscr{B}_{K}$.

We shall now verify that the map $b$ is bijective.

To prove its surjectivity, let us first recall that there exists an elementary solution $E(t, x)$ of the heat operator $(\partial / \partial t-\Delta)[$ i.e., $(\partial / \partial t-\Delta) E(t, x)=\delta(t) \otimes$ $\delta(x)]$ in the space $\mathscr{D}^{\{2\}^{\prime}}\left(\mathbb{R}^{n}\right)$ so that it satisfies

$$
E(t, x)= \begin{cases}(4 \pi t)^{-(n-1) / 2} \exp \left(-x^{2} / t\right), & \text { if } t>0 \\ 0, & \text { if } t<0 .\end{cases}
$$

(Cf. [9])

Now, for a hyperfunction $g(x)$ supported by $K$, we define another hyperfunction $w(t, x)$ by

$$
\iint E(t-s, x-y)(\delta(s) \otimes g(y)) d s d y=\int E(t, x-y) g(y) d y .
$$

Let $w_{+}(t, x)$ denote the restriction of $w$ to $\left\{(t, x) \in \mathbb{R}^{n} ; t>0\right\}$. Then one can easily verify that $w_{+}$satisfies the condition (1). (Cf. [8], Theorem 1. 2) Hence we may consider $b\left(w_{+}\right)$. We shall prove $b\left(w_{+}\right)=g$. For that purpose let us choose a tame extension $v$ of $w_{+}$and set $\mu=(\partial / \partial t-\Delta) v$. [Needless to say, $v$ does not coincide with $w$ in general. $]$ Then it is known $([8]$, p.58) that

$$
v(t, x)=\iint E(t-s, x-y) \mu(s, y) d s d y .
$$

We can further verify that 


$$
\lim _{t \downarrow 0} \int v(t, x) \chi(x) \varphi(x) d x=\int b\left(w_{+}\right)(x) \varphi(x) d x
$$

holds for any entire function $\varphi(x)$, if we choose $\chi(x)$ to be a compactly supported $C^{\infty}$-function which is equal to 1 on a neighborhood of $K$. ([8], (1. 22)) On the other hand, the definition of $w$ entails

$$
\begin{aligned}
\lim _{t \downarrow 0} & \int w_{+}(t, x) \chi(x) \varphi(x) d x \\
& =\lim _{t \downarrow 0} \iint E(t, x-y) g(y) \chi(x) \varphi(x) d x d y \\
& =\int g(x) \chi(x) \varphi(x) d x .
\end{aligned}
$$

(Cf. [8], (1.11)) Since $v(t, x)=w_{+}(t, x)$ holds for $t>0$, the equality $b\left(w_{+}\right)=g$ follows from the denseness of entire functions in $\mathscr{A}(K)$, the space of real analytic functions on $K$. Thus we have verified the surjectivity of $b$.

Finally let us prove the injectivity of $b$. We shall again make use of the elementary solution $E$. Let $v$ be a tame extension of $u$ satisfying the condition (1) and let $\mu(t, x)=\sum_{k=0}^{\infty} \delta^{(k)}(t) \otimes \mu_{k}(x)$ be $(\partial / \partial t-\Delta) v$. (Cf. (11)) Suppose now that $b(u)$ vanishes. Then it follows from the definition of $b(u)$ that $\sum_{k=0}^{\infty} \Delta^{k} \mu_{k}(x)$ vanishes. Hence (26) entails the following relation for $t>0$ :

$$
\begin{aligned}
u(t, x) & =\sum_{k=0}^{\infty} \frac{\partial^{k}}{\partial t^{k}} \int E(t, x-y) \mu_{k}(y) d y \\
& =\sum_{k=0}^{\infty}\left(\frac{\partial^{k}}{\partial t^{k}}-\Delta^{k}\right) \int E(t, x-y) \mu_{k}(y) d y \\
& =\sum_{k=0}^{\infty} Q_{k}\left(\frac{\partial}{\partial t}-\Delta\right) \int E(t, x-y) \mu_{k}(y) d y \\
& =0
\end{aligned}
$$

This proves the injectivity of the map $b$, completing the proof of Theorem 1 .

In the course of the above proof of Theorem 1 (in particular, the part of the proof of the surjectivity of the map $b$ ), we have also verified the following results as by-products. As they seem to have their own interests, we present them as theorems.

Theorem 2. Each function $u$ in $\mathscr{S}_{\mathrm{K}}^{\text {tame }}$ is real analytic.

Theorem 3. For a compactly supported hyperfunction $f(x)$ on $\mathbb{R}^{m}$ we can find 
compactly supported ultradistributions $f_{k}(x)(k=0,1,2, \cdots)$ in $\mathscr{E}^{\{2\}}\left(\mathbf{R}^{m}\right)^{\prime}$ so that

$$
f(x)=\sum_{k=0}^{\infty} \Delta^{k} f_{k}(x)
$$

and

$$
\operatorname{supp} f_{k} \subset \operatorname{supp} f
$$

holds for any $k$.

\section{§2. Miscellaneous Remarks}

In this section we present some remarks on our main results given in the preceding section.

(i) It follows from Theorem 1 that, for a solution $u(t, x)$ of the heat equation that satisfies the condition (1), its "boundary value" $b(u)$ is a well-defined hyperfunction which has the form $\sum_{k=0}^{\infty} \Delta^{k} \mu_{k}(x)$ with $\mu_{k}(x)$ being an ultradistribution determined by a tame extension of $u$. Now the following question naturally arises: What if $u(t, x)$ grows faster than $\exp (\varepsilon / t)$ as $t \downarrow 0$ ? In this case we can still find an extension $v$ of $u$ if we allow $u$ to be a hyperfunction. However the series $\sum_{k=0}^{\infty} \Delta^{k} \mu_{k}(x)$ given in an analogous way as in the proof of Theorem 1 does not define a hyperfunction in general. This explains why Aronszajn [1] needed a class of generalized functions that is bigger than the space of hyperfunctions when he discussed the boundary value of a solution of the heat equation.

(ii) In connection with the above remark, we note that we can prove the following

Theorem 4. Let $K$ be a compact set in $\mathbf{R}^{n-1}$. Then for each $\mu(t, x)$ in $\mathscr{E}_{\{0\} \times K}\left\{\mathbf{R}^{n}\right)^{\prime}$ we can find $v(t, x) \in \mathscr{B}_{\{0\} \times K}$ and $g(x) \in \mathscr{B}_{K}$ so that

$$
\mu=(\partial / \partial t-\Delta) v+\delta(t) \otimes g(x)
$$

holds. Furthermore $\nu$ and $g$ are uniquely determined by $\mu$.

In fact, the existence of $v$ and $g$ can be verified in exactly the same manner as in the proof of (15). Since the Fourier transform of $v$ and that of $g$ are both entire functions, the uniqueness assertion can be readily verified if we apply the Fourier transformation to the relation (30).

(iii) The same result as Theorem 1 holds if $\Delta$ is the Laplace operator on a compact Riemannian manifold $M$ (without boundary) and if we consider the heat equation on $\mathbf{R}_{t} \times M$ and choose $M$ as $K$. 


\section{References}

[1] Aronszajn, N., Preliminary notes for the talk "Traces of analytic solutions of the heat equation", and Trace of analytic solution of the heat equation. Astérisque, 2 et 3 (1973), 5-34 and 35-68.

[2] Friedman, A., Partial Differential Equations of Parabolic Type, Prentice-Hall, Englewood Cliffs, N.J., 1964.

[3] Kawai, T., On the theory of Fourier hyperfunctions and its applications to partial differential equations with constant coefficients, J. Fac. Sci. Univ. Tokyo, Sect. IA, 17 (1970), 467-517.

[4] Komatsu, H., Ultradistributions, I; Structure theorems and a characterization, J. Fac. Sci. Univ. Tokyo, Sect. IA, 20 (1973), 25-105.

[5] - Ultradistributions, II; The kernel theorem and ultradistributions with support in a submanifold, J. Fac. Sci. Univ. Tokyo, Sect. IA, 24 (1977), 607-628.

[6] - Introduction to the Theory of Generalized Functions. Iwanami Shoten, Tokyo, 1978. (In Japanese)

[7] Komatsu, H. and T. Kawai, Boundary values of hyperfunction solutions of linear partial differential equations, Publ. RIMS, Kyoto Univ., 7 (1971-72), 95-104.

[8] Matsuzawa, T., A calculus approach to hyperfunctions I, Nagoya Math. J., 108 (1987), 5366.

[9] - Hypoellipticity in ultradistribution spaces, J. Fac. Sci. Univ. Tokyo, Sect. IA, 34 (1987), 779-790.

[10] - A calculus approach to hyperfunctions, II, to appear in Trans. Amer. Math. Soc.

[11] Schapira, P., Théorème d'unicité de Holmgren et opérateurs hyperboliques dans l'espace des hyperfonctions, An. Acad. Brasil. Ci., 43 (1971), 39-44. 